\title{
Competências interculturais de aprendentes chineses no contexto português de imersão: uma investigação qualitativa multidimensional
}

\author{
Hanzi Zhang \\ Universidade de Estudos Estrangeiros de Tianjin \\ zhang.hanzi@yahoo.com
}

\section{Resumo}

A partir de uma perspectiva de educação intercultural no ensino-aprendizagem de PLE, o presente estudo aborda o enquadramento das dimensões (atitude, conhecimento, aptidões, consciência) de competências interculturais, na perceção dos aprendentes chineses de PLE no seu estudo de imersão. Com base na análise dos dados da investigação qualitativa, foi permitido chegar às seguintes conclusões: 1) a perceção do público-alvo chinês sobre CI tem como base um modelo multidimensional composto pelas dimensões acima enunciadas; 2) na codificação dessas dimensões verifica-se no público-alvo chinês a existência de uma diferenciação equilibrada no que se refere à identificação e resolução de dificuldades/problemas; 3) no contexto de imersão em Portugal, vê-se a valorização de atitudes e da consciência intercultural e constata-se a lacuna do conhecimento e o défice linguístico e não-linguístico nas aptidões do público-alvo chinês.

Palavras-chave: competências interculturais; aprendentes chineses; PLE; imersão.

\section{Abstract}

Based on the intercultural education perspective in the teaching and learning of Portuguese as Foreign Language, the present study intends to address the framework of dimensions of intercultural competences (attitudes, knowledge, skills and awareness), in the perception of Chinese learners of Portuguese in their study of immersion. The analysis of qualitative research data allows us to arrive at the following conclusions: 1) the perception of the Chinese learners about $\mathrm{CI}$ is based on a multidimensional model composed by those dimensions; 2 ) in the codification of dimensions, there is a marked and balanced differentiation in the Chinese learners by the identification of difficulties and the resolution of difficulties; 3 ) in the immersion context in Portugal, it's obvious the valuation of attitudes and awareness and the lack of knowledge and skills, mainly due to the linguistic and 
non-linguistic deficit of language learners.

Keywords: intercultural competences; Chinese learners; PLE; immersion.

\section{Introdução}

Acompanhando o crescente interesse em aprender a língua portuguesa, têm surgido discussões no que diz respeito à formação dos falantes chineses em língua portuguesa, com o intuito de alcançar uma melhor qualificação, melhor competência comunicativa e um melhor desenvolvimento pessoal. Desse modo, em sinergia com a tendência evolutiva da educação em nível internacional, cada vez mais universidades chinesas elaboraram, nos últimos anos, protocolos com universidades portuguesas que permitem o intercâmbio de alunos de licenciatura (ZHENG, 2010).

No ensino-aprendizagem de Potuguês Língua Estrangeira (PLE), passar um ano em contexto de imersão representa para os aprendentes chineses uma extensão da aprendizagem, o que gera um avanço significativo para eles, muito além do que é normalmente desenvolvido nas instituições educacionais, como explana Murphy-Lejeune (2003) na defesa do intercâmbio educacional. Porém, percorrendo o estado de arte na literatura de educação intercultural (FANTINI, 2000; COULBY, 2006; SERCU, 2006), chama-se a atenção para o facto de que ao expor os alunos nas experiências internacionais, sem os preparar adequadamente ou sem os apoiar para tomarem consciência das suas experiências, podem aparecer resultados negativos de aprendizagem, que refletem vulnerabilidades tanto nos alunos como nos programas de intercâmbio. Mesmo no seguimento dessas observações, politicamente explicitadas ou observadas a partir da experiência, a resposta dada pelas instituições nem sempre é satisfatória. Deardorff (2004) identifica que há poucas instituições de ensino superior que procuravam abordar a questão de desenvolvimento de estudantes interculturalmente competentes como um resultado antecipado de internacionalização, na qual o conceito de "competência intercultural" (doravante designada por CI) é especificamente definido, e ainda menos instituições que, efetivamente, designavam métodos para documentar e medir a competência intercultural. 


\subsection{Questões da investigação}

Este trabalho não pretende ser excessivamente ambicioso na formulação das perguntas de investigação. As perguntas a que se procura responder ao longo do estudo são:

1.1.1 Qual é ou quais são as dimensões na percepção sobre a CI entre os aprendentes chineses de PLE em contexto de imersão?

1.1.2 Qual é ou quais são os choques culturais nos encontros interculturais entre os aprendentes chineses de PLE em contexto de imersão?

\section{Definição de CI}

São inúmeros autores que têm vindo a contribuir com perspetivas variadas na procura de uma definição de CI (DEARDORFF, 2004), o que levou a que não se tenha ainda conseguido um consenso nem na definição, nem na terminologia em torno de CI (DEARDORFF, 2006; 2011). Após a revisão de literatura na área, Fantini (2006) recolhe uma variedade dos termos utilizados, referindo-se tanto ao estado da arte como aos instrumentos de avaliação. Entre elas, destacam-se os termos cross-cultural awareness, global competitive intelligence, cultural competence, cultural sensitivity, ethno-relativity, international competence, intercultural interaction, biculturalism, multiculturalism.

Ao entrarmos na abordagem de definição do termo CI, não é uma tarefa fácil identificar qual é ou quais são os pontos de partida para a elaboração da questão. Os assuntos que devem ser levados em conta são, por exemplo: a CI deve ser definida de maneira mais geral ou mais específica? O que constitui a CI essencial (DEARDORFF, 2004)? Como pode a CI ser avaliada (LUSTIG; KOESTER, 2003)? O que deveria ser realmente aprendido (BOWDEN; MARTON, 1998)? No nosso trabalho, será feito um enquadramento das definições na área, sobretudo aquelas que são mais reconhecidas e cujas perspetivas nos conseguem orientar para construir uma retrospeção do desenvolvimento concetual e disciplinar de CI, em mais de quatro décadas, tendo sempre em consideração a complexidade do próprio tema do estudo.

Uma das primeiras definições de CI vem de Tewksbury, que criou uma lista de 21 "Characteristics of a Mature International Person" em 1957 (WILSON, 1994, p. 41-42). Desde então, Robert Hanvey (1976) fornece 
uma definição de CI bastante citada, que inclui cinco dimensões interdisciplinares da educação global: perspective consciousness, state of the planet awareness, cross-cultural awarness, systemic awareness e options for paticipation. Entre essas dimensões, a consciência do estado de planeta e a consciência intercultural estão intimamente relacionadas com o que hoje em dia chamamos de CI. Nesse aspecto, o pressuposto teórico de constituição por três componentes fundamentais de Spitzberg e Cupach (1984) é provavelmente um dos mais influentes no estudo de conceito de CI. Os três componentes são: a motivação, o conhecimento e as habilidades. Gudykunst (1994) vem reafirmar a importância da motivação, ao lado de conhecimento e de habilidades que compõem o essencial da CI. Guo-Ming Chen e William J. Starosta (1996) definem a competência comunicativa intercultural como "the ability to negotiate cultural meanings and to execute appropriately effective communication behaviors that recognize the interactants' multiple identities in a specific environment” (p. 358-359). Trata-se de uma afirmação que valoriza a interação eficaz e apropriada entre os interlocutores interculturalmente competentes, em que são indispensáveis a sensibilidade afetiva, a consciência cognitiva e a habilidade comportamental.

À volta da controvérsia da conceitualização de CI, surgem imensas dificuldades na abordagem do tema, à luz de exemplo, está a avaliação de muitos programas vocacionados para o treinamento intercultural (CHEN; STAROSTA, 2000). Porém, depois do olhar panorâmico e do enquadramento de CI ao longo desses anos, apesar das visões epistemológicas às vezes conflituosas dos estudos conduzidos, sobretudo nas décadas 70 e 80, por autores diferentes, de perspetivas diversas e com objetivos distintos, testemunha-se um certo consenso entre os autores em termos ao contorno das definições de CI. Tomando como exemplos, a proposta dos componentes como a motivação, o conhecimento e as habilidades de Spitzberg e Cupach (1984), de Gudykunst (1994) e de Spitzberg (1997); a relevância dada na definição de Chen e Starosta (1996) e de Bennett e Bennett (2003) nas habilidades de atingir "effectiveness" e "appropriateness"; o conhecimento, as aptidões, a atitude e a consciência crítica internacional, que estão presentes na modelização de Byram (1997), acompanhando a aquisição de língua estrangeira no contexto europeu; a associação inclusiva de quatro dimensões: o conhecimento, as aptidões, a atitude e a consciência com o "effectiveness" nas competências comunicativas e o "appropriateness" nas competências cognitivas, na 
perceção de Fantini (2000); a interdependência entre as competências cognitivas, as afetivas e as praxeológicas assenta na própria natureza indissociável entre as três componentes, de acordo com Kim (2005); o contexto interpessoal e situacional, o grau de adequação e efetividade de interação, assim como o conhecimento, a motivações e as ações suficientes, bastante enfatizados por Lustig e Koester (2003) ao utilizar o termo CI; as dimensões individuais compostas pelos conhecimento, compreensão, aptidões e atitude formam paralelamente o modelo pirâmide de comunicação intercultural com as dimensões interativas, assim se interpreta por Deardorff $(2004,2006)$.

As teorizações acima referidas revestem-se como base de fundamentos para a formulação de prismas conscientes dos documentos da União Europeia e da UNESCO, que, respetivamente, objetivam, sem nenhuma ambiguidade, a CI dos cidadãos no mundo de globalização. No documento Developing intercultural competence through education (2014), a CI é vista como "a combination of attitudes, knowledge, understanding and skills applied through action which enables one, either singly or together with others" (p. 16), enquanto que a UNESCO (2013) aponta a CI como as habilidades que permitem a cada indivíduo ser capaz de navegar em ambientes complexos, marcados por uma crescente diversidade de povos, de culturas e de estilos de vida.

Recapitulando, ressaltam-se dois elementos que são repetidamente referidos pela maior parte dos autores na concetualização de CI: o contexto intercultural e o "effectiveness and appropriateness" e, na discussão deles sobre as dimensões constituintes de CI, o conhecimento, a aptidão, a atitude e a consciência são aqueles que ganham uma posição dominante, como ilustra na figura 1:

Figura 1. Composição de CI

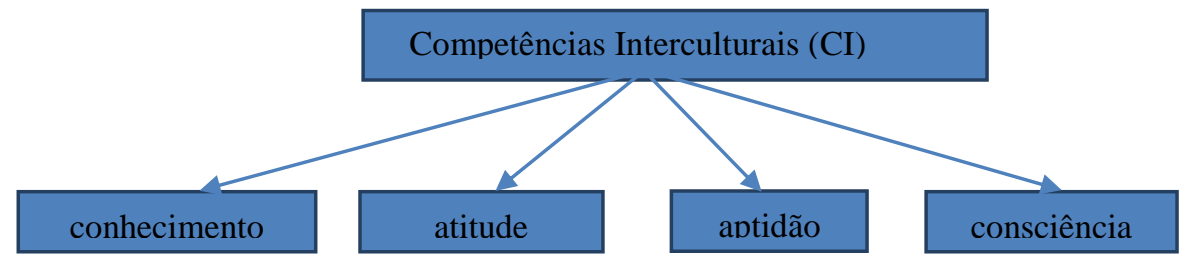

Fonte: Fantini $(2000 ; 2006)$ 


\section{Metodologia}

\subsection{Realização da investigação}

Perante a plena expansão dos projetos e acordos bilaterais de intercâmbio de alunos, o número dos alunos chineses nos últimos anos atingiu um número sem precedentes, nas instituições portuguesas e brasileiras do ensino superior. À luz das considerações sobre os objetivos e o público-alvo chinês da investigação, que apontam para esses alunos em imersão, o presente estudo opta por selecionar como participantes no inquérito os alunos chineses que estão neste momento no contexto de imersão português. O Autobiography of Intercultural Encounters ${ }^{1}$ foi adaptado e administrado em língua portuguesa, através do correio eletrónico, entre os dias 24 e 31 de março de 2018. No total, foram recolhidas 20 respostas válidas, que se processam a seguir à análise qualitativa de dados.

\subsection{Análise dos dados}

Após a administração do instrumento de investigação, as respostas foram diretamente introduzidas no programa Nvivo 11.0 e posteriormente processadas e analisadas também neste programa. Os resultados serão apresentados e discutidos na próxima seção. Fundamentada em grounded theory (STRAUSS; CORBIN, 1990) e em qualitative content analysis (SCHREIER, 2012), a análise dos dados procura, sistematicamente, descrever o significado dos dados qualitativos, atribuindo-se ao corpus um quadro de categorias com codificações. Em primeiro lugar, uma leitura pormenorizada do corpus é feita de mente aberta, de modo a permitir o surgimento e o ressurgimento das palavras originais. Através dessas palavras originais, fazem-se a comparação e a agregação até se identifica a sua categorização, noutra palavra, a codificação. Na sequência da codificação preliminar, procede-se à codificação temática na base de leitura repetitiva sobre os dados e assim se apresenta na Tabela 1 na subseção 4.2, um exemplo em que se vislumbra o processo de codificação e classificação.

\footnotetext{
${ }^{1}$ https://www.coe.int/t/dg4/autobiography/default_en.asp, acedido a 29 de maio de 2018.
} 


\section{Análise e leitura dos dados}

\subsection{Análise descritiva}

Dentro do tamanho de 200-300 palavras, verifica-se que em cada uma das respostas, em função do tema a ser exposto, foi utilizado um ou dois géneros na escrita. Entre os temas revelados, o foco do público-alvo chinês no contexto português de imersão linguística e cultural, destacam-se as conversas sobre as tradições, as preferências e os gostos que diferenciam uma cultura de outra, bem como os encontros com a população local, as atividades escolares e extraescolares e o desenvolvimento de entendimento próprio sobre a interação intercultural.

De uma forma geral, os choques culturais mais comuns encontram-se nas diferenças de tradições, de gostos e de preferências entre as duas culturas, sobretudo o que há em uma e não há em outra. A título de exemplo, é considerado difícil a experiência nos primeiros contatos, em particular, aceitar dar beijinhos como forma de cumprimento em Portugal. A falta de pontualidade e de planejamento com antecedência também são apontados e constituem umas outras complicações para o público chinês aceitar e interagir com sucesso. A acrescer a isso, pelas descrições na investigação, é possível identificar o desconhecimento ou o preconceito da parte do comunicador português no que diz respeito às políticas internas na China, o que faz com que as duas partes de comunicação não consigam estabelecer uma atitude positiva um com o outro logo no primeiro contato. A timidez e a hesitação de articular oralmente as suas ideias, da parte do público chinês, em larga medida também se afiguram como obstáculos para a comunicação intercultural e até para desenvolver a CI. Porém, em muitos casos, as características apresentadas durante os encontros pelos portugueses, como a hospitalidade,a extroversão e até idiossincrasias específicas, são apreciados e facilitam o sucesso de encontro.

Após esta caraterização geral do corpus, na subseção seguinte, será analisada qualitativamente as incidências em torno da avaliação de CI. Lembra-se que, para garantir a autenticidade do próprio corpus, os erros e inadequações linguísticas ocorridos são mantidos na análise e leitura dos dados e na redação do presente trabalho. 


\subsection{Análise qualitativa}

Na subseção presente, debruçamo-nos sobre a análise qualitativa do conteúdo do corpus, designadamente as 139 incidências que conseguimos verificar em torno à avaliação de CI pelo nosso público-alvo e que se procedem ao método de análise de acordo com as justificativas na subseção acima. A título de exemplo, segue-se o quadro 1 como um excerto de categorização das incidências do corpus.

Quadro 1. A categorização exemplificativa das codificações

\begin{tabular}{|l|l|l|}
\hline Nível 1 & Nível 2 & Nível 3 \\
\hline $\begin{array}{l}\text { "Respeitar a cultura totalmente diferente" } \\
\text { "ele ajuda me tanto numa maneira tão simpática" }\end{array}$ & Atitude & \\
\hline $\begin{array}{l}\text { "Eles deixaram-me a pensar em algo que eu } \\
\text { nunca tinha pensado" } \\
\text { "a perceber que às vezes não há } \\
\text { comportamentos bons, maus, estranhos, apenas p } \\
\text { orque somos diferentes, somos das culturas diver } \\
\text { sas" }\end{array}$ & Consciência & Resolução \\
$\begin{array}{l}\text { "Eu não percebi" } \\
\text { "não sabia quando devia se beijar" } \\
\text { "sinto-me menos confusa às ações dos nativos" }\end{array}$ & Conhecimento & \\
\cline { 1 - 2 } "Porém não podia fazer nada" & Dificuldade \\
"não sabia o que devia falar" & Aptidão & \\
\hline
\end{tabular}

Fonte: Elaborado pelo autor

No resultado analítico da investigação, em jeito de síntese, delineia-se um panorama diferenciado pela orientação por identificação de dificuldades/problemas (69 ocorrências) e pela orientação por resolução de dificuldades/problemas (70 ocorrências). A divisão equilibrada entre a identificação e a resolução de dificuldades interculturais oferece-nos umas pistas que vislumbram a tendência do público, que ao mesmo tempo expõem as adversidades encontradas, procuram abordá-las pressupondo as soluções possíveis. Aquando das codificações subjacentes das orientações acima mencionadas, detetamos nos autorrelatos do público-alvo chinês, com bastante clareza e em sinergia com o enquadramento dos fundamentos teóricos da maior parte dos autores da área, as quatro dimensões de CI: a atitude, a consciência, o conhecimento e as aptidões. 


\subsubsection{Atitude}

Em larga medida, o público-alvo chinês apresenta uma atitude positiva em relação à população portuguesa, que, pela sua natureza, demonstra uma afeição em relação aos interlocutores que são provenientes de outra cultura, o que ajuda na quebra de gelo em muitos casos de comunicação. Isto revela-se pelas incidências como "que interessante", "me sentia a empatia", "ela agradou-me", "hospitalidade dos portugueses", "a paixão dos portugueses", "o entusiasmo e a extroversão", "ela era tão simpática", entre outros. De maneira esta, a mesma afeição transmitida pelos comunicadores portugueses traduz-se na reformulação da própria atitude do público-alvo chinês, uma vez que "we may show respect towards them, meaning we see value and positive elements in their beliefs and practices even if we do not share them" (CONSELHO EUROPEU, AIE, p. 4). E se se considerar a base de CI estar nas atitudes das pessoas que interagem com as de outra cultura (INCA, p. 9), torna-se ainda mais relevante a reciprocidade de atitude positiva e de respeito entre os envolventes numa comunicação intercultural.

Em contraponto, no desenrolar de encontros, também se depreendem os traços atitudinais que complexificam a comunicação. Exemplos desses são como "estava super-supreendida e mesmo zangada", "fiquei irritada", "me sentia sozinha", "tinha receio", "a minha timidez", entre outros. Tratam-se de reações que, de forma negativa, apareceram logo nos primeiros contatos de encontros e, geralmente, são as respostas que se dão com "prejudice, suspicion and intolerance" (CONSELHO EUROPEU, AIE, p. 4), tanto da parte de orador como da parte de interlocutor.

A aceitação do Outro e a salvaguarda de direitos do Outro, mesmo que não esteja de acordo com ele, pode-se traduzida como "tolerance" (CONSELHO EUROPEU, AIE, p. 4). No que se concerne ao respeito na comunicação, Deardorff (2004) indica a importância de demonstrar que os outros são valorizados. Neste aspeto, o público-alvo propõe resoluções como, por exemplo, "com base de respeito mútuo", "não esperamos tanto", "tolerar o mais possível", "aceitar as diferenças culturais", "os meus sentimentos naquele tempo eram curiosidade", "ela estava aberta à pessoa desconhecida, dava-me a paixão preciosa", e "nós apresentamos respeito um ao outro, portanto recebemos carinho das desconhecidas". 


\subsubsection{Conhecimento}

Em primeiro lugar, o melhor conhecimento sobre uma cultura parte do entender todas as experiências que norteiam os seus membros ao longo de vida (JANDT, 2010). No âmbito do presente trabalho, não será abordada a discussão de definições de cultura (Ver: SPENCER, 2008); este estudo centra-se maioritariamente nas manifestações exteriores de cultura que se relacionam visualmente com o corpus sobre os encontros interculturais. As incidências deste género são como "quando iria apertar as mãos com ela, ela veio e beijou nas minhas bochechas", "não sabia quando devia se beijar"; "as tradições não mudam", "o gosto dos portugueses é bem diferente com o gosto dos chineses", "os portugueses são os cavaleiros de aventura e os chineses têm os pés fincados na terra", etc. Além disso, o conhecimento sobre a cultura e as práticas culturais, da sua própria cultura de origem e da cultura de imersão, não só implica as práticas, as crenças, os valores, os símbolos, as tradições e as maneiras de ser e de interpretar o mundo (CONSELHO EUROPEU, AIE), mas também preconiza a compreensão sobre a língua e o gesto, a aparência pessoal, a religião, os costumes familiares, a comida, o trabalho, o sistema de educação e de comunicação, os sistemas de saúde, de transporte e de governo, entre outros (JANDT, 2010).

Percorrendo o corpus, não é difícil de localizar aqui e ali as ocorrências de "desacordos", os casos "estranhos" e "inesperados", de estar surpreendidos, de muitas vezes "nunca tinha pensado", o que transparece uma lacuna de conhecimento do nosso público em relação às práticas e mentalidade culturais de Portugal e o caminho indispensável para o progresso de competências comunicativa e intercultural do nosso públicoalvo.

\subsubsection{Aptidões}

Por se tratar de aprendentes da língua no contexto de imersão, o nosso público, de modo geral, refere que nos encontros interculturais as comunicações decorreram via língua portuguesa, como tal, as competências comunicativa e sócio comunicativa da língua portuguesa surgiram como as aptidões mais apontadas para o sucesso do encontro. Exemplos desses são "através da comunicação", "foi uma comunicação interessante e significada", "a comunicação entre dois línguas diferentes também foi uma 
experiência especial". No tocante à competência comunicativa, uns impedimentos e dificuldades também são descritos como "não tínhamos maneiras para comunicarmos", "devo comunicar mais com ela" e assim por diante.

Tomando como o foco do nosso estudo as CI, as aptidões em causa têm uma abrangência mais estendida. Hammer, Gudykunst e Wiseman (1978) sugerem "the ability to manage psychological stress, the ability to communicate effectively, and the ability to establish interpersonal relationships" (p. 206). Com base no trabalho deles, Pusch (1994; 2009) amplia um pouco a terceira de Hammer, Gudykunst e Wiseman (1978), nomeadamente, a habilidade de aproveitar a interface entre culturas e o conhecimento que nasce de diferentes orientações culturais e a habilidade de administrar as mudanças, à medida que as fronteiras nacionais se dissolvem e as culturas se encontram imediatamente por meio de tecnologia (PUSCH, 2009). Pelo que se mostra no corpus, constantemente, para o público chinês falta esta aptidão de administrar bem o stresse e as mudanças circunstanciais, as expressões como "eu senti-me um pouco nervoso" e "tinha receio" constituem a prova de isto. Outras aptidões que permitem a interação eficaz e apropriada, no momento de encontro, também se encontram em ausência, com o público reclamando que "não podia fazer nada", "não é nada fácil de mudar", "não sabia o que devia falar", "não conseguia procurar uma maneira adequada para eu praticar bem a língua portuguesa". Muitas vezes, pela ausência das aptidões em função de estabelecer relacionamentos, administrar as disfunções e mediar entre duas culturas, o objetivo formativo de "intercultural speaker" no ensino-aprendizagem de LE, pegando a designação de Byram (1997), pode não ser atingido. Para que isso não seja uma realidade, devem ser levadas em conta e acionadas as aptidões de CI a partir de observar, escutar, avaliar, analisar, interpretar e relacionar (DEARDORFF, 2004), até à atitude e ao conhecimento que abordamos anteriormente como a base de progresso de aptidões.

Segundo a nossa descoberta, as maneiras mobilizadas pelo nosso público são como "tentei explicar", "divertimos jogos juntos", fazer a "amizade", no entanto, continuam a ser insuficientes para ir ao encontro ao que Byram (1997) define para as aptidões de CI. 


\subsubsection{Consciência}

Ao se fazer a revisão da literatura dos estudos na consciência de CI, descobre-se que Chen e Starosta estão entre os autores que são mais citados e mais produções científicas têm. Os dois autores (1998) opinam que a consciência intercultural é a perspetiva cognitiva da comunicação intercultural e, na perceção deles, a consciência intercultural vem salientar a relevância do conhecimento cultural. Chen e Starosta (1998) consideram esta perspetiva cognitiva (consciência intercultural) como o fundamento de "intercultural sensitivity" que, por sua vez, conduz à componente praxeológica de CI. Kohls (1996) também realça a autoconsciência (consciência da própria bagagem cultural) como a mais necessária, que serve de sustentação para entender e interagir efetivamente com os outros, provenientes de origens diferentes. Pela análise feita, o nosso público já está ciente da distância e a diferença que separam a cultura portuguesa da cultura de origem, que neste caso é a chinesa, o que é bastante favorável à construção mais profunda de consciência intercultural, tanto no que se refere às semelhanças como também sobre as diferenças. Uma declaração encontrada no corpus diz-se assim: “... a perceber que às vezes não há comportamentos bons, maus, estranhos, apenas porque somos diferentes, somos das culturas diversas". É por meio dos encontros que estão em causa e de muitos outros encontros que não foram registados, mas ocorreram no dia a dia, que o público chinês desenvolve a sua consciência intercultural. As incidências como "foi a primeira vez que tive este tipo de experiência", "esta experiência pôs-me a pensar em algo que nunca tinha pensado", "esta frase fazia-me pensar muito" atestam a sensibilização que tem ocorrido durante estes encontros.

A respeito de poder ter maior consciencialização cultural do nosso público, as observações indicam "considerar mais a diferença cultural" ou "ter mais cuidado e paciência em tomar decisão", perante o insucesso de encontro, não obstante a formação continuada e ainda mais esforçada neste aspeto. As alternativas, porém, estão na mão de professores, que podem não apenas desenvolver a consciência de CI, mas sim a sua consciência crítica em geral (CHICK, 1996). 


\section{Discussão e conclusões}

Partindo de uma questão muito pertinente do ensino-aprendizagem de PLE de hoje e com o público que gera um crescimento contínuo de procura de português, o presente estudo visa vislumbrar a perceção dos aprendentes chineses de PLE no seu estudo de imersão, no domínio especializado em competência intercultural. A retrospetiva da história da evolução desta área de investigação, em torno de definições, modelos e taxonomias de CI, permite delinear um enquadramento teórico dos estudos de CI, chegando a um vazio a ser colmatado, que solicita uma perspetiva não-ocidental e feita a partir dos aprendentes. Para possibilitar uma maior coerência em termos de fundamentos teóricos com os estudos congéneres, o presente estudo adotou e modificou um instrumento replicável de investigação qualitativa, nomeadamente o Autobiography of Intercultural Encounters, do Conselho Europeu e aplicou-o a uma amostra do público-alvo chinês. A análise e a leitura dos dados, no seu conjunto e as análises dimensionais, permitem-nos chegar às seguintes conclusões: 1) a perceção do público-alvo chinês sobre CI está assente em categorização e modelos multidimensionais compostos por Atitude, Conhecimento, Aptidão e Consciência; 2) na codificação superior de dimensões, verifica-se no público-alvo chinês a diferenciação equilibrada da orientação por identificação de dificuldades/problemas e por resolução de dificuldades/problemas, o que permite presumir que a aprendizagem de CI do público-alvo chinês é como um processo de identificação e resolução; 3) no contexto de imersão em Portugal, por um lado, a atitude positiva recíproca entre os aprendentes da língua e os falantes nativos favorece a interação intercultural, assim como a consciencialização do público-alvo no tocante às semelhanças e diferenças. Por outro lado, constata-se a lacuna do conhecimento devido à distância cultural e a défice linguística e não-linguística nas aptidões do público-alvo chinês.

Em referência à lacuna detetada no presente estudo, trata-se de conhecimento e de aptidões do público chinês que poderão ser alimentados por formação educativa constante, paralelamente à aprendizagem de PLE. Byram (1997) sugere três espaços de aquisição de CI, respetivamente nos contextos de "classroom", "fieldwork" e "independent learning" (p. 38). Enquanto o Conselho Europeu (2014) aconselha "experiential learning or learning by doing", "project work" e "co-operative learning" (p. 37). As experiências, as comparações, as análises, as reflexões, as tarefas baseadas 
em tópicos ou em temas, são exemplos de caminhos por explorar que, neste caso, ficam nas mãos dos professores e administradores de programas internacionais.

Estas conclusões vêm confirmar a pertinência e a importância de formação de CI dos aprendentes chineses da língua portuguesa, além da competência comunicativa. Além disso, os resultados da investigação qualitativa no público que está em imersão, sugerem que existem especificidades neste contexto de aprendizagem, tal como aponta vários caminhos de trabalho para os ensinantes e investigadores, que devem conhecer esta realidade de maneira a poder haver novas mudanças pedagógicas. Porém, o presente estudo não está isento de limitações. $\mathrm{O}$ estudo é, sem dúvida, um esforço preliminar. De uma perspetiva sustentável, o corpus de 20 respostas válidas deste estudo foi relativamente modesto, não sendo considerado suficiente para chegar a uma conclusão generalizada. Os objetivos deste estudo levaram à realização de uma investigação qualitativa. Percebeu-se, também, que a combinação de investigações qualitativa e quantitativa fornece os dados mais confiáveis, do ponto de vista dos aprendentes ou do ponto de vista dos ensinantes. Por último, a partir dos resultados do presente estudo, também se julga importante investigar como será efetuado o ensino-aprendizagem da CI, para além de PLE, nas aulas da língua, ou como será feito o desenho dos currículos ou dos programas de intercâmbios interinstitucionais.

\section{Referências}

BARRETT, M. D.; HUBER, J.; REYNOLDS, C. Developing intercultural competence through education. Strasbourg: Council of Europe Publishing, 2014.

BOWDEN, J.; MARTON, F.. The university of learning. London: Kogan Page, 1998.

BENNETT, J. M.; BENNETT, M. J. Developing intercultural sensitivity. In: LANDIS, D.; BENNETT, J.; BENNETT, M. (Ed.). Handbook of intercultural training. London: Sage Publications, 2003. p. 147-165.

BYRAM, M.. Teaching and assessing intercultural communicative 
Competências interculturais de aprendentes chineses...

competence. Clevedon: Multilingual Matters, 1997.

COULBY, D. Intercultural education: theory and practice. Intercultural Education, v. 17, n. 3, p. 245-257, 2006.

CHEN, G.-M.; STAROSTA, W. J. Intercultural communication competence: a synthesis. Communication Yearbook, v. 19, p. 353-383, 1996.

CHEN, G.-M.; STAROSTA, W. J. A review of the concept of intercultural awareness. Human Communication, v. 2, p. 27-54. 1998/9.

CHEN, G.-M; STAROSTA, W. J. The development and validation of the intercultural sensitivity scale. Human Communication, v. 3, p. 1-15, 2000.

CHICK, J. K. Intercultural communication. In McKay, S.L.; Hornberger, N. H. (Eds.), Sociolinguistics and language teaching. Cambridge: Cambridge University Press, 1996. p. 329-348.

CONSELHO EUROPEU. Autobiography of intercultural encounters. 2009. Disponível em: <https://www.coe.int/t/dg4/autobiography/Source/AIE_en/AIE_concepts_f or_discussion_en.pdf $>$. Acesso em: 24/10/2017.

DEARDORFF, D. K. The identification and assessment of intercultural competence as a student of international education at institutions of higher education in the United States [dissertation]. Unpublished dissertation, University of North Carolina, Raleigh, North Carolina. 2004.

DEARDORFF, D. K. Identification and assessment of intercultural competence as a student outcome of internationalization. Journal of studies in international education, v. 10, n. 3, p. 241-266, 2006.

DEARDORFF, D. K. Assessing intercultural competence. New directions for institutional research, v. 149, p. 65-79, 2011.

FANTINI, A. E. A central concern: Developing intercultural competence. SIT occasional papers series, v. 1, p. 25-42, 2000. 
FANTINI, A. E. Exploring and assessing intercultural competence. Brattleboro, VT: Federation of the Experiment in International Living. 2006. Disponível em <http://www.worldlearning.org/ 7803.htm or http://www.experiment.org/resources.html>. Acesso em 08 mar. 2018.

GUDYKUNST, W. B. Bridging differences: Effective intergroup communication. Thousand Oaks, CA: Sage Publications, 1994.

HAMMER, M. R.; GUDYKUNST, W. B.; WISEMAN, R. L. Dimensions of intercultural effectiveness: An exploratory study. International Journal of Intercultural Relations, v. 2, n. 4, p. 382-393, 1978.

HANVEY, R. G. An attainable global perspective. New York: Global Perspectives in Education. 1976.

JANDT, F. E. An introduction to intercultural communication: Identities in a global community. Thousand Oaks, CA: Sage Publications, 2010.

KIM, Y. Y. Inquiry in intercultural and development communication. Journal of communication, v. 55, n. 3, p. 554-577, 2005.

KOHLS, L. R. Survival kit for overseas living ( $3^{\text {rd }}$ ed.). Yarmouth, ME: Intercultural press, 1996.

LUSTIG, M. W.; KOESTER, J. Intercultural competence: Interpersonal communication across cultures ( $4^{\text {th }}$ ed.). Boston: Allyn \& Bacon. 2003.

MURPHY-LEJEUNE, E. An experience of interculturality: Student travellers abroad. In: Alfred, A.; Byram, M.; Fleming, M. (Eds.), Intercultural experience and education. Clevedon: Multilingual Matters, 2003. p. 101-113.

PUSCH, M. D. The chameleon capacity. In: Lambert, R. D. (Ed.) Educational exchange and global competence. New York: Council on Intercultural Educational Exchange, 1994. p. 205-210.

PUSCH, M. D. The interculturally competent global leader. In: Deardorff, D. K. (Ed.) The SAGE handbook of intercultural competence. Thousand 
Competências interculturais de aprendentes chineses...

Oaks, CA: Sage Publications, 2009. p. 66-84.

SERCU, L. The foreign language and intercultural competence teacher: The acquisition of a new professional identity. Intercultural education, v. 17, n. 1, p. 55-72, 2006.

SCHREIER, M. Qualitative content analysis in practice. London: Sage Publications, 2012.

STRAUSS, A.; CORBIN, J. M. Basics of qualitative research: Grounded theory procedures and techniques. Thousand Oaks, CA: Sage Publications, 1990.

SPITZBERG, B. H. Intercultural effectiveness. In: Samovar, L. A. \& Porter, R. E. (Eds.) Intercultural communication: A reader. Bermont, CA: Wadsworth, 1997. p. 379-391.

SPITZBERG, B. H.; CUPACH, W. R. Interpersonal communication competence. Beverly Hills, CA: Sage Publications, 1984.

UNESCO; LEEDS-HURWITZ, W.; STENOU, K. Intercultural Competences: Conceptual and Operational Framework. UNESCO: Intersectoral Platform for a Culture of Peace and Non-Violence, 2013.

WILSON, A. H. The attributes and tasks of global competence. In: Lambert, R. D. (Ed.), Educational exchange and global competence. New York: Council on International Educational Exchange, 1994. p. 37-50.

ZHENG, S. O ensino da língua portuguesa na China: Caracterização da situação actual e propostas para o futuro (Dissertação de Mestrado). Universidade do Minho. 2010.

Submetido em: 30/07/2018 Aceito em: 20/12/2018

Title: Intercultural competences of Chinese learners in the Portuguese context of immersion: a qualitative multidimensional investigation 